Moreno Pizarro, J. \& Planells Hernani, B. (2016). Propuesta para la implementación de la teoría de las Inteligencias Múltiples en el sistema de Educación Infantil en España. Revista Electrónica Interuniversitaria de Formación del Profesorado, 19 (3), 199-207.

DOI: http://dx.doi.org/10.6018/reifop.19.3.267341

\title{
Propuesta para la implementación de la teoría de las Inteligencias Múltiples en el sistema de Educación Infantil en España
}

Jessica Moreno Pizarro, Begoña Planells Hernani

Universidad Camilo José Cela

\section{Resumen}

El enfoque de la presente propuesta se centra en la importancia que tienen las Inteligencias Múltiples. El objetivo de este trabajo es conseguir un cambio en la metodología didáctica que actualmente se plantea en el sistema educativo español de educación infantil.

Integrar las inteligencias múltiples a través del juego, como base del proceso educativo es sin lugar a dudas un gran apoyo a la docencia y proporciona a la enseñanza-aprendizaje una de las herramientas necesarias para fomentar el trabajo colaborativo que proporcionan los juegos.

Es por ello necesario incluir cambios en la forma de enseñar, para que se produzcan cambios en la forma de aprender; el maestro deberá ser un guía que trabaje en colaboración con los alumnos.

Se programará una didáctica específica pensada para trabajar en un centro escolar en el que la metodología será tradicional, en la que se tendrán en cuenta las características personales de cada uno de los alumnos, así como, las necesidades educativas específicas, planteando una óptica multidimensional, perfilando los distintos campos del talento.

\section{Palabras clave}

Inteligencias múltiples; trabajo colaborativo; juegos educativos; talento. 


\section{A proposal for the implementation of the multiple intelligence theory in the Spanish early childhood education system}

\section{Abstract}

This research proposal focuses on the importance of the multiple intelligences.

The aim of this paper is to make a change in the teaching methodology currently applied in the Spanish early childhood education system.

Integrating multiple intelligences through the game, as the basis of the educational process, is undoubtedly a great teaching support and provides with one of the necessary tools to promote collaborative work that games offer.

It is therefore necessary to make changes in the teaching methodology, so that the way we learn will also be changed; the teacher should be the guide to work in collaboration with students.

A specific teaching programme will be designed to work in a school where the methodology is traditional, considering the personal characteristics of each student, as well as the specific educational needs posing a multidimensional perspective and outlining the various fields of talent.

\section{Key words}

Multiple intelligences; educational cooperation; education games; talent.

\section{Introducción}

El contexto en el que nos encontramos socialmente requiere una renovación e innovación pedagógica, es por ello que en esta propuesta que pretendemos implementar en el futuro se plantea una propuesta de cambio, valorando el modelo actual de la Didáctica que se lleva a cabo en Educación Infantil. Quizás con esta propuesta se establezca un punto de inflexión, requiriendo trabajar de una forma más práctica, para adaptar y encaminar este programa como una realidad en el sistema educativo de educación infantil a través de las Inteligencias Múltiples (Gardner, 1995).

La futura propuesta, pretende llevar a cabo la elaboración de un modelo didáctico, así como estimular las distintas inteligencias en el aula de educación infantil, destacando la importancia de una educación personalizada de los alumnos, para obtener una mayor motivación e interés por los contenidos de cada materia a través del juego contemplando la posibilidad de la incorporación de las nuevas tecnologías. Hacemos especial hincapié en la atención a la diversidad porque ésta supone un medio de enriquecimiento. Aprender va unido a hacerlo en grupo, se facilita la tarea a todos los alumnos y se mejora la calidad educativa. A través del trabajo cooperativo en las aulas, y con el juego como principal medio para descubrir, crear, fomentar la motivación, incrementa la confianza de los alumnos, por tanto constituye, una metodología basada en todo un desafío a la Creatividad y a la Innovación en el Sistema Educativo. 
Integrar las inteligencias múltiples a través del juego, como base del proceso educativo es sin lugar a dudas un gran apoyo a la docencia y proporciona a la enseñanza-aprendizaje una de las herramientas necesarias con las cueles el alumnado no solo trabaja a su propio ritmo, sino que también se fomenta el trabajo colaborativo que proporcionan los juegos, potenciando estos aún más este proceso de aprendizaje.

El cambio didáctico se basará en la evaluación de las capacidades individuales, tomando como referencia la teoría de las inteligencias múltiples propuesta por Gardner (1999) y sus estudios sobre el aprendizaje llevado a la práctica destacando la importancia de la estimulación de los dos hemisferios cerebrales, la parte lógica y la parte creativa.

Dado que es una propuesta para la implementación futura, se pretende la instauración de este nuevo modelo didáctico que facilitará a todos los estudiantes alcanzar el rendimiento académico adecuado, en base a estrategias concretas de aprendizaje, apropiadas y semejantes con las capacidades de éstos, entendiéndose como tal el concepto de inteligencia, todo ello a través de una propuesta para la aplicación de las Inteligencias Múltiples en el aula, incluyendo el juego en la etapa de educación infantil como una herramienta de aprendizaje global. Es por ello necesario incluir cambios en la formar de enseñar, para que se produzcan cambios en la forma de aprender; por ello el maestro deberá ser un guía que trabaje en colaboración con los alumnos.

Concretamente, se programará una didáctica específica pensada para trabajar en un centro escolar en el que la metodología será tradicional, con una dependencia excesiva de los libros, un uso reducido de nuevas tecnologías, una aplicación limitada del aprendizaje cooperativo y en el que la lección magistral es el eje fundamental.

En esta propuesta se tendrán en cuenta las características personales de cada uno de los alumnos, así como, las necesidades educativas específicas. Para tener un conocimiento previo de la inteligencia que destaca en cada uno de los niños se llevarán a cabo la realización de test de Inteligencias Múltiples de Gardner (1983), actividades previas y juegos.

Howard Gardner, en su obra Frames of Mind (1983), definió la inteligencia humana como la capacidad de resolver problemas o crear cosas reconocidas por la uno o varios ambientes culturales. Pero la mayor aportación a la definición de inteligencia, que desarrolló Gardner, es llevar esta definición, no solo al ámbito lingüístico y lógico matemático, sino que amplía el concepto hacia una naturaleza pluralista del potencial cognitivo. También hace referencia a la importancia de la interacción de los factores biológicos y ambientales.

A partir de la concreción de 8 tipos distintos de desarrollo inteligente independientes entre sí. A partir de su aportación de las IM, plantea una óptica multidimensional, perfilando los distintos campos del talento.

A continuación veremos la clasificación de las 8 inteligencias que propuso (Gardner, 1983, 1995, 2001). 


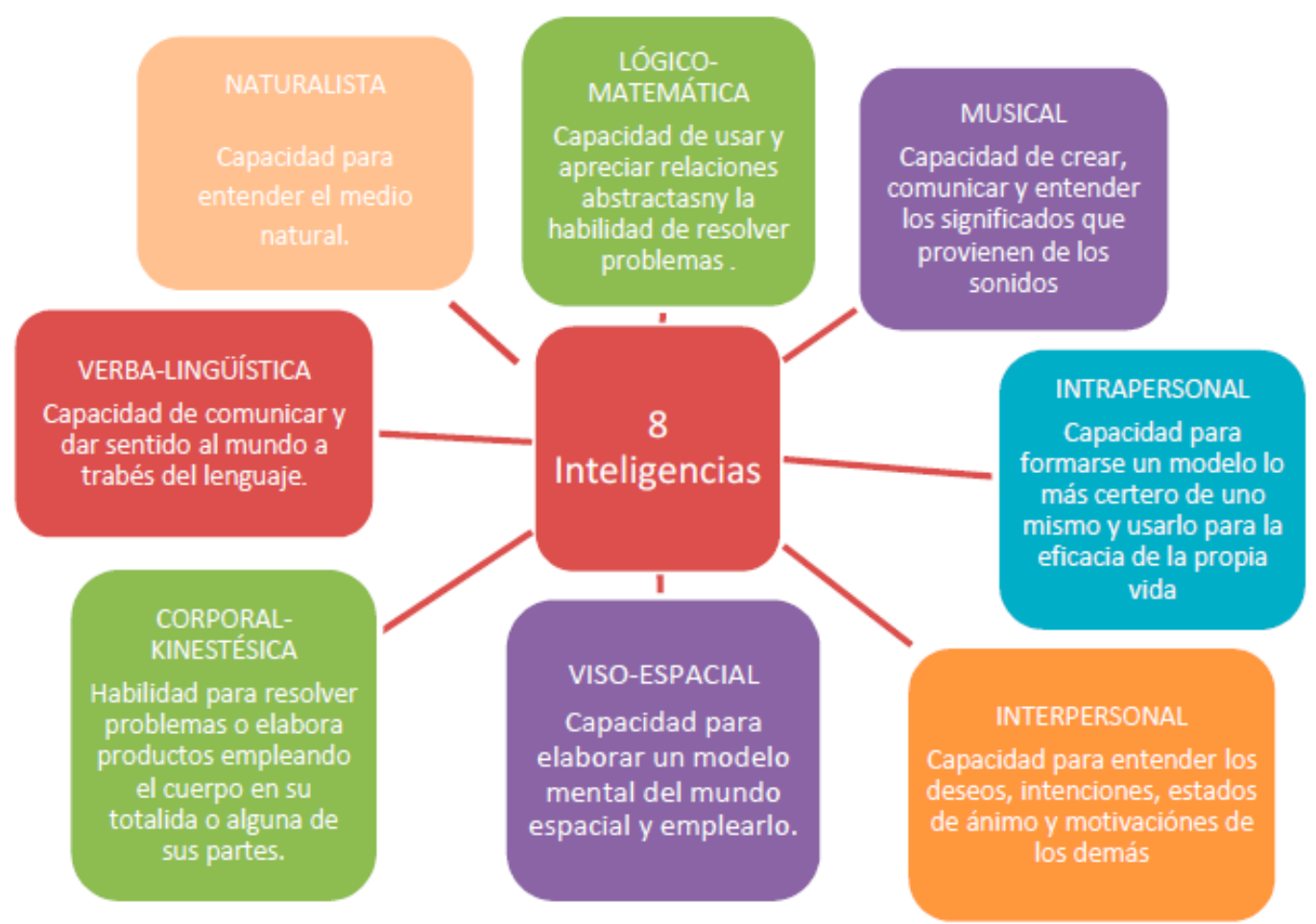

Figura 1. Cuadro de la propuesta de Gardner de las Inteligencias Múltiples, (Prieto y Castejón, 2000)

\section{Propuesta de trabajo}

Esta unidad, llamada "Enséñame a aprender", está pensada para implementarla durante el primer trimestre. Se compone de siete bloques de espacios y se realizarán dos veces por semana, cada sesión durará 1 hora. El alumnado trabajará por equipos los cuales rotarán por dos espacios, 20 minutos por espacio, de tal modo que al final de la sesión todos los alumnos habrán realizado dos de las actividades propuestas, y al finalizar la semana todos nuestros alumnos habrán pasado por todos los espacios. Al finalizar cada sesión se dedicarán diez minutos a presentar y/o explicar lo que han visto, así como lo que han aprendido y como han resuelto posibles problemas.

Nuestra propuesta se ajusta al siguiente esquema (Figura 2):

La acción educativa de los equipos de trabajo, es una propuesta metodológica. Lo que propone es delimitar diferentes espacios diferenciados dentro del aula, donde los alumnos puedan realizar de manera simultánea diferentes actividades de tipo cognitivo, manipulativo y simbólico. Estos espacios concretos desarrollan diferentes valores y alternativas para conseguir unos objetivos, contenidos, hábitos y actitudes. Y con ello que aprendan a observar, manipular, experimentar, etc. 


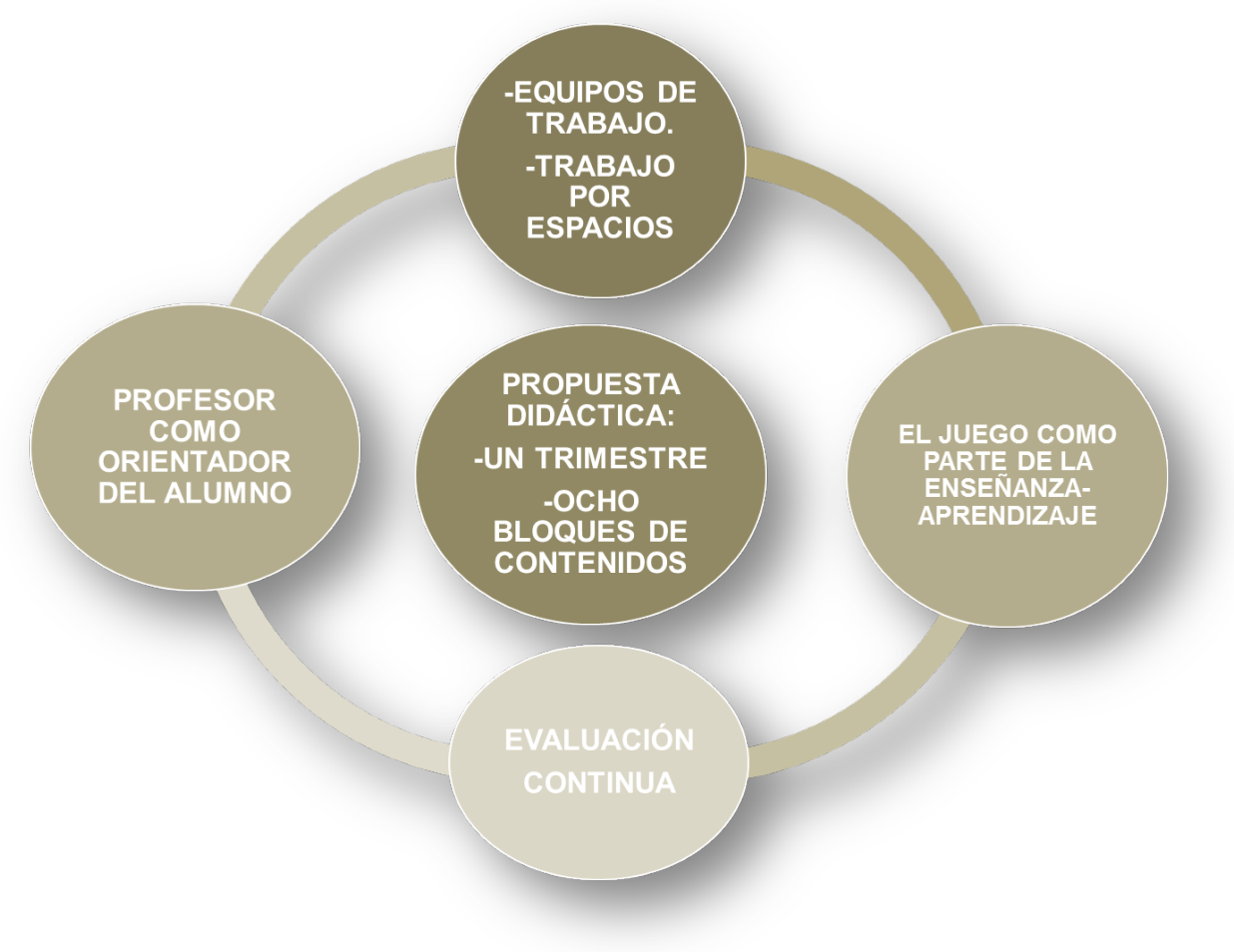

Figura 2. Implantación de la propuesta "Enséñame a aprender".

Los equipos de trabajo provocan la necesidad y el deseo de aprender, así como de investigar nuevas técnicas y estrategias para resolver problemas y favorece la autonomía. Se debe ofrecer una variedad de propuestas a realizar para que los alumnos puedan incorporarse de manera libre y autónoma a cada uno de los espacios que se planteen.

Es una propuesta de trabajo abierto y flexible, el rol del profesor es guiar, observar y dinamizar los aprendizajes de cada alumno.

Organizar la clase por equipos implica conocer bien al grupo-clase, sus necesidades, inquietudes y dificultades, con las que se va a trabajar, crear un buen clima, de confianza, de tranquilidad. El juego es el principal instrumento de aprendizaje que tienen los alumnos, cuando un alumno está jugando en equipo está trabajando, disfrutando y adquiriendo unos conocimientos.

Implicaciones de la metodología por equipos de trabajo

La metodología por equipos de trabajo implica tener muy presente los siguientes aspectos:

- Aceptar la diversidad del alumnado. Cada alumno tiene su ritmo madurativo, tiene sus necesidades y dificultades. Ofrecer una gran variedad de actividades, permite llevar a cabo una atención más personalizada de los alumnos/as.

- Potenciar las diferentes inteligencias impulsando el uso de los diferentes lenguajes (musical, matemático, lingüístico, plástico, etc).

- Aconsejar a los alumnos para que tengan un criterio propio y puedan elegir el que más les guste o les apetezca realizar.

- Marcar tiempos de interrelación, donde todos los alumnos tengan las mismas oportunidades de manipular y conocer todo el material disponible. 
- Considerar al alumnado como agente activo en sus aprendizajes a través de los sentidos y de la manipulación.

- Observar las posibles dificultades que tiene cada uno de los alumnos en los distintos espacios, así como en los equipos de trabajo y ofrecerles la ayuda pedagógica que necesiten.

Antes del comienzo del trabajo a realizar, dependiendo del número de alumnos que haya en el aula, les indicaremos a los alumnos cuántos equipos deben formar y cuantas personas pueden componer cada equipo.

En este momento el profesor deberá acompañar y guiar a los alumnos, pero debe dejarlos libertad en todo momento para la elección a realizar.

Es importante que cada día que se realiza esta actividad los grupos de trabajo serán distintos a los del día anterior.

Formados los equipos de trabajo es en ese momento, será cuando los alumnos y el profesor se sientan y presentan el material que hay en cada uno de los espacios que se han creado en el aula de forma concreta. A continuación, se explica lo que hay que hacer o las posibilidades que ofrece cada uno.

Una vez que se comience a trabajar por equipos de trabajo, es importante recordar las normas.

Tabla 1.

Cronograma de Las actividades de la Propuesta Didáctica (Elaboración propia)

\begin{tabular}{|c|c|c|c|}
\hline & JUEGOS DE APRENDIZAJE & INTELIGENCIAS MÚTLIPLES & $\begin{array}{l}\text { COMPETENCIAS } \\
\text { BÁSICAS }\end{array}$ \\
\hline \multirow{4}{*}{$\begin{array}{l}1^{\mathrm{a}} \\
\text { semana }\end{array}$} & Memory (imagen-palabra) & 1,3 y 7 & 1 y 8 \\
\hline & Uno (juego de cartas) & 2 y 7 & 2 y 8 \\
\hline & Experimentamos con la cámara & 3,6 y 8 & $3,4,5,6$ y 7 \\
\hline & Componemos una canción & 4 y 6 & 4 y 5 \\
\hline \multirow{4}{*}{$\begin{array}{l}2^{\mathrm{a}} \\
\text { semana }\end{array}$} & Bingo (sumas y restas) & 2,3 y 7 & 2 \\
\hline & Buscamos palabras & 1 y 7 & 1 y 8 \\
\hline & $\begin{array}{l}\text { Autorretrato a partir de la imagen del } \\
\text { espejo }\end{array}$ & 3 y 6 & 3 \\
\hline & Hacemos fotos & 3,6 y8 & $3,4,5$ y 6 \\
\hline \multirow{4}{*}{$\begin{array}{l}3^{a} \\
\text { semana }\end{array}$} & Elaboramos un menú & 1 y 3 & 1 y 8 \\
\hline & Domino de sumas y restas & 2 y 6 & 2 y 8 \\
\hline & Mímica & 5.6 y 7 & 1,4 y 8 \\
\hline & Hacemos fotos & 3,6 y 8 & $3,4,5$ y 6 \\
\hline \multirow{3}{*}{$\begin{array}{l}4^{\mathrm{a}} \\
\text { semana }\end{array}$} & Tangram & 2, y 3 & 2 y 7 \\
\hline & Títeres & 1,5 y 7 & 1 y 8 \\
\hline & Descubrimos la calculadora & 1 y 8 & 2 y 7 \\
\hline
\end{tabular}




\begin{tabular}{|c|c|c|c|}
\hline & Hacemos fotos & 3,6 y8 & $3,4,5$ y 6 \\
\hline \multirow{4}{*}{$\begin{array}{l}5^{a} \\
\text { semana }\end{array}$} & Memory de instrumentos en tablet & 3 y 4 & 3,5 y 6 \\
\hline & Simón dice & 5 y 7 & 4 y 8 \\
\hline & Pictionary & 3 y 7 & 3 y 8 \\
\hline & Hacemos fotos & 3,6 y 8 & $3,4,5$ y 6 \\
\hline \multirow{4}{*}{$\begin{array}{l}6^{a} \\
\text { semana }\end{array}$} & Restaurante & 1,2 y 7. & $1,2,4,6,7$ y 8 \\
\hline & Buscando la pareja & 1 & 7 \\
\hline & Secuencias & 1 y 2 & 1 у 2 \\
\hline & Hacemos fotos & 3,6 y8 & $3,4,5$ y 6 \\
\hline \multirow{4}{*}{$\begin{array}{l}7^{a} \\
\text { semana }\end{array}$} & Preparamos la exposición & 3,6 y 7 & 4,7 y 8 \\
\hline & ¿Cuál ha sido nuestra experiencia? & 1 у 2 & 1,3 y 4 \\
\hline & Tienda & 1,2 y 3 & 1,6 y 8 \\
\hline & ¿Quién es quién? & 1 y 3 & 1 y 8 \\
\hline
\end{tabular}

Tabla 2.

Cuadro explicativo de la tabla 1. (Elaboración propia)

\begin{tabular}{|c|c|}
\hline Inteligencias & Competencias \\
\hline 1. Inteligencia lingüístico-verbal. & $\begin{array}{l}\text { 1. Competencia comunicativa, lingüística } \\
\text { audiovisual. }\end{array}$ \\
\hline 2. Inteligencia lógico-matemática & 2. Competencia matemática \\
\hline 3. Inteligencia viso-espacial & 3. Competencia artística y cultural. \\
\hline 4. Inteligencia musical. & 4. Competencia de autonomía e iniciativa \\
\hline 5. Inteligencia corporal-cinestésica. & $\begin{array}{l}\text { 5. Tratamiento de la información y competencia } \\
\text { digital }\end{array}$ \\
\hline 6. Inteligencia interpersonal. & $\begin{array}{l}\text { 6. Competencia en el conocimiento y la interacción } \\
\text { con el mundo }\end{array}$ \\
\hline 6. Inteligencia Intrapersonal. & 7. Competencia de aprender a aprender \\
\hline 7. Inteligencia naturalista. & 8. Competencia social y ciudadana \\
\hline
\end{tabular}

Por otro lado, se decide el espacio por el que va a empezar cada uno de los equipos. Es importante tener una tabla de doble entrada donde quede registrado el nombre de los alumnos y espacio de trabajo elegido.

A continuación vemos el registro que llevará el profesor:

Tabla 3.

Distribución de los Juegos De Aprendizaje (Elaboración propia). 


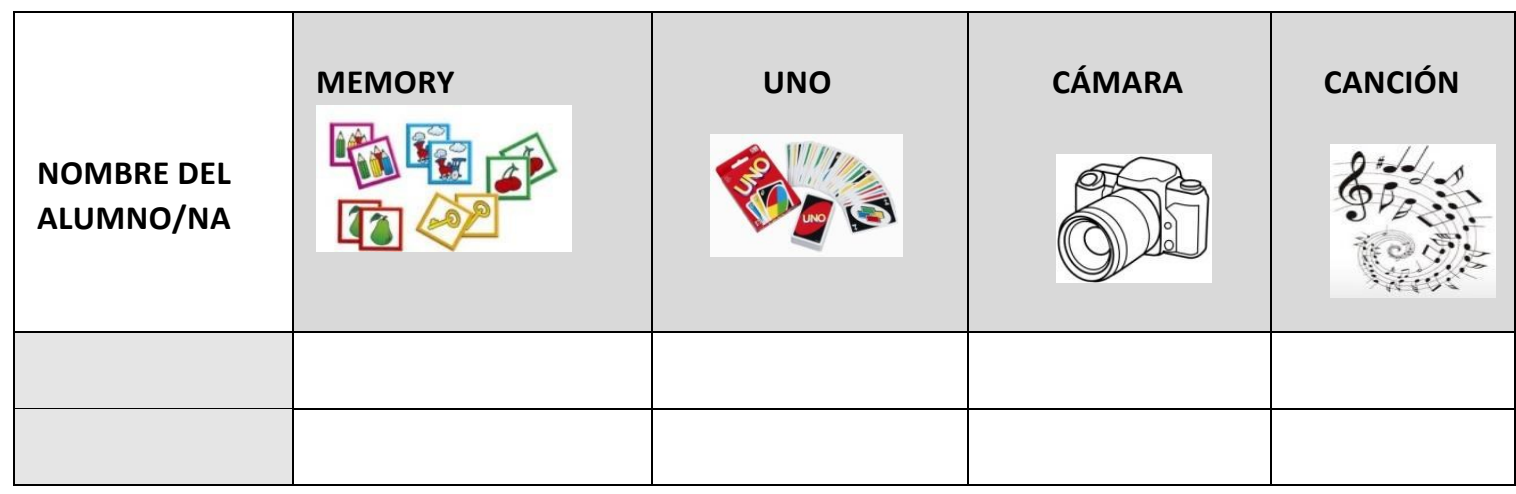

En cuanto a la evaluación de esta propuesta educativa, es evidente que no se pueden establecer líneas de actuación rígidas ni desarrollar un proceso de evaluación sistemático. Es por ello que, consideramos la evaluación desde tres enfoques diferentes pero que a la vez se complementan y enriquecen entre sí mismos; inicial para comprobar el nivel educativo del alumnado; continua para asegurarnos del proceso de enseñanza aprendizaje; y por último sumativa para llevar a cabo una valoración global de cada discente (Bloom, Hastings, Madaus, 1971; 1975).

\section{Discusión y conclusiones}

Los motivos que impulsaron el desarrollo del programa han sido de diverso tipo. Por una parte, mejorar el proceso de enseñanza - aprendizaje, ya que desde hace años han revelado que las peculiaridades del alumnado son diversas, y en el día a día del aula resulta complejo poder atender al alumnado de forma individual. Por otro lado, se tendrá en cuenta que podrá ser favorable para el alumnado trabajar todos los contenidos desde diversas áreas, ya que los aprendizajes se verán reforzados y el alumnado podrá aplicar los conocimientos y hacerlos más duraderos.

A lo largo de los últimos cursos, se ha demostrado que los niños muestran dificultad para transmitir los conocimientos alcanzados de un contexto. Esta limitación es debida a la imitación con la que se presentan los contenidos con frecuencia.

Es de vital importancia tener en cuenta que los cambios producidos en toda institución educativa son intencionados y premeditados, con el objetivo de cambiar el sentido de una situación en función de las necesidades que se hayan observado y puestas de manifiesto, a partir del conocimiento de los problemas que aquejan a los sujetos implicados en el proceso educativo. La implementación en el sistema educativo español, quizás no sea tan rápida, recordando que siempre vamos cinco años por detrás de las nuevas tecnologías.

Por otro lado, establecer escuelas piloto y ofrecer el método a nivel privado, podría servir de base, para que los políticos del futuro puedan implementar esta estructura educativa en el Sistema de Educación Infantil en España.

Si todo lo anterior se alcanza, podremos garantizar una educación creativa, flexible, con eficacia, sin exclusión y con un alto rendimiento. Una didáctica planificada para un individuo del siglo XXI, en donde las inteligencias múltiples puedan ser desarrolladas al máximo y con ello podamos obtener una sociedad realmente eficaz. 


\section{Referencias}

Cambpell, L. (2000). Multiple Intelligences and Student. Achievement: Success Stories from Six Schools. Success Stories from Six Schools. USA.

Bloom, B. S., Hastings, T., y Madaus G. (1975). Evaluación del aprendizaje. Buenos Aires: Troquel.

García Hoz, V. (1988). Tratado de educación personalizada. Práctica de la educación personalizada. Madrid: Rialp.

Gardner, H.(1983). La Estructura de la mente. Barcelona: Paidós.

Gardner, H. (1995). Inteligencias múltiples. La teoría en la práctica. Barcelona: Paidós.

Gardner, H. (1998). Inteligencias múltiples. La teoría en la práctica. Barcelona: Paidós.

Gardner, H. (2001): La inteligencia reformulada. Las inteligencias múltiples en el siglo XXI. Barcelona: Paidós. 